\title{
Reviewers 2019
}

\author{
K. Abbot-Smith \\ S. Allen \\ F. Altinkamis \\ C. Alvarez \\ J. Arciuli \\ S. Arunachalam \\ S. Babayigit \\ V. Bambini \\ N. Banasik-Jemielniak \\ C. Bannard \\ D. Barner \\ E. Barnes \\ R. Barr \\ I. Barriere \\ D. Beals \\ L. Bedore
}

M. Beeghly

H. Behrens

T. Benders

C. Bergmann

P. Bernardini

S. Bernolet

R. Blything

H. Boelens

H. Bortfeld

M. Braginsky

S. Brandt

J. Bresnan

S. Brouwer

E. Bruno

P. Brusini

H. Buckler

A. Bunger

A. Bus

K. Byers-Heinlein

T. Cameron-Faulkner

A. Carmiol

J. Carpendale

S. Carroll

M. Casillas

Y. S. Chan
M. Charest

J. Chen

V. Chondrogianni

E. Clark

P. Cleave

J. Cleland

C. Contemori

C. Core

L. Cote-Reilly

S. Creel

A. Cristia

J. Culbertson

S. Curtin

P. Dale

L. Davidson

C. Davies

L. de Almeida

B. DeBaryshe

A. De Houwer

J. de Jesus

J. de Jong

S. de la Rie

H. de Mulder

R. de Paolis

J. de Villiers

K. Demuth

S. Dettman

D. Dickinson

H. Diessel

J. Dijkstra

G. Dionne

M. Dittmar

J. Dockrell

B. Dodd

G. Dove

W. Dressler

L. Duchesne

S. Durrant

J. Dynia

M. Erbuagh

J. Evers-Vermeul
L. Fabiano-Smith

L. Farran

M. Fasolo

C. Félix-Brasdefer

E. Ferstl

P. Fletcher

C. Flores

A. Foltz

S. Foster-Cohen

F. Franco

V. Freeman

S. Fricke

P. Ganea

H. Ganek

J. Ganger

A. Garcia-Sierra

M. Garraffa

E. Gavruseva

S. Geffen

F. Genesee

D. Gentner

J. Gerard

R. Gillam

S. Gillis

L. Gogate

T. Göksun

A. Goldberg

R. Golinkoff

N. Gonzalez-Gomez

D. Gooch

H. Goodluck

J. Goodman

J. Goodrich

A. Goodwin

S. Graham

J. Grinstead

J. Gros-Louis

T. Grueter

E. Haman

C. Hamman

A. Hammer

(C) Cambridge University Press 2019 


\begin{tabular}{|c|c|c|}
\hline E. Hannon & H. Littlefield & J. Patterson \\
\hline N. Havron & C. Lukyanenko & A. Pellicer-Sanchez \\
\hline He Sun & E. Lund & F. Perek \\
\hline J. Heilmann & I. Lundeborg Hammarström & A. Perez-Leroux \\
\hline H. Hendriks & R. Luo & L. Pierce \\
\hline C. Herve & L. Lustigman & J. Pine \\
\hline T. Hickey & B. MacWhinney & M. A. Pinto \\
\hline M. Hickmann & A. McCabe & A. Plumb \\
\hline T. Hills & L. McCune & M. Polinsky \\
\hline E. Hoff & D. McDaniel & K. Polisenka \\
\hline B. Höhle & M. McGillion & B. Pomiechowska \\
\hline H. Норp & M. Majorano & C. Potter \\
\hline A. A. Howard & V. Marchman & P. Prevost \\
\hline I. Hübscher & V. Marrero & L. Price \\
\hline C. Hudson Kam & G. Martohardjono & J. Punske \\
\hline H. Hwang & P. Meara & J. Quach \\
\hline N. Hyams & N. Meir & N. Ramirez-Esparza \\
\hline P. Ibbotson & C. Mesa & M. Ramscar \\
\hline J. Iverson & D. Miller & D. Ravid \\
\hline M. Jasbi & U. Minai & L. Raviv \\
\hline Jing Lin & G. Mirabella & C. Read \\
\hline G. Jones & S. Mishina-Mori & K. Read \\
\hline M. Kalashnikova & P. Mok & V. Reddy \\
\hline L. Kapa & P. Monaghan & C. Reuterskiold \\
\hline W. Katz & S. Montrul & P. Rinaldi \\
\hline E. Kay-Raining Bird & J. Morgan & J. Rispens \\
\hline M. Kehoe & G. Morini & M. Roberts \\
\hline S. Kennison & V. Mueller Gathercole & P. Rollins \\
\hline T. Keren-Portnoy & K. Mulak & R. Romeo \\
\hline E. Kidd & I. Mundy & M. Rosenberg \\
\hline M. Kilani-Schoch & L. Naigles & G. Rost \\
\hline M. Kirjavainen-Morgan & K. Nakamura & M. Rowe \\
\hline T. Kokkinaki & C. Narayan & S. Rvachew \\
\hline B. Köpke & D. Nelson & E. Saiegh-Haddad \\
\hline A. Krott & E. Nguyen & N. Salerni \\
\hline S. Kucker & E. Nicoladis & V. Salo \\
\hline L. Laks & A. Nicolopoulou & L. Santelmann \\
\hline E. Lau & D. Nielsen & J. Schaeffer \\
\hline C. Lawson & A. Ninio & A. Schick \\
\hline M. Le Normand & M. Nippold & P. Schneider \\
\hline J. Lee & I. Nomikou & E. Schoen Simmons \\
\hline K. Leech & A. Nyhout & F. Schwarz \\
\hline G. Legendre & C. O’Madagain & L. Serratrice \\
\hline J. Lepola & J. Oakhill & P. Shimpi \\
\hline D. Levine & K. Oatley & L. Shneidman \\
\hline F. $\mathrm{Li}$ & M. Ota & A. Shusterman \\
\hline Li Liu & A. Pace & C. Silvey \\
\hline J. Liceras & A. Papafragou & D. Simard \\
\hline E. Lieven & D. Pascual y Cabo & D. Slobin \\
\hline
\end{tabular}



V. Smith
C. Snow
S. Sonnerschein
D. Sperry
M. Spinelli
A. Stöhr
V. Stojanovik
S. Stokes
S. Stolt
Y-C. Su
D. Suskind
T. Suzuki
G. Szagun
S. Tal
C. Tamis LeMonda
T. Tardif
M. Taumoepeau
A.-M. Tessier

R. Tincoff

X. S. Tong

K. Tonsing

L. Tsung

K. Twomey

Y. Uchikoshi Tonkovich

V. Valian

D. Valois

M. van Dam

M. van Puyvelde

M. Vasilyeva

C. Venker

V. Vihman

C. Volman

M. Wagers

H. Waterfall

S. Wauquier

A. Wedel
A. Weisleder

R. Weist

J. Whalen

K.White

B. Woll

B. Wolter

Xi Chen

Xiangjun Deng

M. Yavaş

S. Yeung

Yi-Ching Su

Yi Ting Huang

V. Yip

H. Yoshida

C. Yoshinaga-Itano

Q. Yow

Yuanyuan Wang

P. Zydorowicz 